\section{Evaluation of the Sensitivity and Specificity of a Commercially Available Rapid Syphilis Test - Escambia County, Florida, 2016}

James Matthias ${ }^{1,2}$; Patty Dwiggins ${ }^{3}$; Yolanda Totten ${ }^{4}$; Carina Blackmore ${ }^{2}$; Craig Wilson ${ }^{2}$; Thomas A. Peterman ${ }^{1}$

In December 2014, the Food and Drug Administration granted the first-ever Clinical Laboratory Improvement Amendments waiver for a rapid treponemal syphilis screening test, Syphilis Health Check (SHC) (1). SHC is a new tool for public health programs to combat increasing syphilis rates, specifically among persons without a prior syphilis infection. SHC can be performed by nonlaboratorian health care personnel and results are available in 10 minutes. In 2015, a total of 7,094 noncongenital cases of syphilis $(35.8$ case per 100,000$)$ were reported to the Florida Department of Health (2). The Florida Department of Health evaluated the performance of SHC in comparison with treponemal and nontreponemal tests routinely used in its sexually transmitted disease (STD) clinic in Escambia County.

For this evaluation, patients seeking STD testing at the Florida Department of Health STD clinic in Escambia County during March 11-April 21, 2016, were tested for syphilis using the SHC on blood specimens obtained by fingerstick; a venous blood specimen was drawn concurrently and submitted for treponemal (Trep-Sure), and nontreponemal (Arlington Scientific, Inc. [ASI] rapid plasma reagin [RPR] card test for syphilis) testing at the state public health laboratory. The state public health laboratory in Florida uses the CDC-recommended algorithm for syphilis testing (i.e., nontreponemal testing followed by treponemal testing for persons with a reactive nontreponemal test); however, for the purpose of this study, all collected specimens underwent treponemal testing regardless of the nontreponemal test result. The SHC result was compared with results of routine syphilis testing using the traditional testing algorithm at the state laboratory. Sensitivity, specificity, and overall laboratory test agreement were determined using the Trep-Sure qualitative enzyme immunoassay (EIA) reference treponemal test as the standard for "true" positive or negative treponemal test results.

The SHC was used to screen 202 patients for syphilis. Among these patients, $171(85 \%)$ were nonreactive on all syphilis tests (SHC, EIA, and RPR), 26 (13\%) had a reactive SHC, and five (2\%) had a nonreactive SHC but had one or more reactive tests at the state laboratory. Among the 26 reactive SHCs, 10 (38\%) had a reactive EIA (six had a reactive RPR), and 16 (62\%) were not confirmed by EIA or RPR at the state laboratory. For the six reactive $\mathrm{SHC}$ patients with reactive EIA and reactive RPR, three were staged as secondary syphilis, one as primary syphilis, one as early latent syphilis, and one was a previously treated positive with no increase in titer since last testing. Among the five specimens that were reactive on other tests but $\mathrm{SHC}$ nonreactive, only one was both RPR (1:8 serum dilution) and EIA reactive. It came from a patient with primary syphilis and a history of herpes simplex virus 2 , and a reactive $\mathrm{RPR}$ (1:2 serum dilution) that was collected 6 days before the SHC test.

The sensitivity of SHC was $71.4 \%$ (95\% confidence interval $[C I]=41.9 \%-95.1 \%)$ when compared with the Trep-Sure (EIA) reference treponemal test (Table). The specificity of the SHC compared with the reference treponemal test was $91.5 \%$ $(95 \% \mathrm{CI}=87.5 \%-95.5 \%)$.

The findings in this study are subject to at least one limitation. The sample size was 202; however, results indicate a high proportion of reactive SHC tests were not confirmed by reference treponemal testing (16 of 26, 61.5\%). This relatively low positive predictive value suggests that reactive $\mathrm{SHC}$ results should be interpreted with caution. Furthermore, four of 14 specimens that tested positive on the reference treponemal test tested negative on the SHC, including one from a patient with primary syphilis. Sensitivity and specificity analyses of the SHC using fingerstick specimens at the Florida Department of Health in Escambia County's STD clinic were significantly lower than the $>98 \%$ reported by the manufacturer of SHC in a $510(\mathrm{k})$ submission (3). Further evaluation of the sensitivity and specificity of the SHC in additional health care settings is needed to determine whether SHC might be beneficial in identifying patients who might have syphilis, especially in settings where phlebotomy is unavailable.

TABLE. Comparative results of Syphilis Health Check testing of specimens $(\mathrm{N}=202)$ at the Florida Department of Health in Escambia County STD clinic and Trep-Sure reference treponemal testing at the state health department laboratory - Florida, 2016

Trep-Sure (EIA) result

\begin{tabular}{lcc}
\cline { 2 - 3 } Syphilis Health Check result & Reactive & Nonreactive \\
\hline Reactive (26) & 10 & 16 \\
Nonreactive (176) & 4 & 172 \\
Total (202) & 14 & 188
\end{tabular}

Testing agreement $\%(95 \% \mathrm{Cl})$

\begin{tabular}{ll}
\hline Sensitivity* & $71.4(41.9-95.1)$ \\
Specificity* $^{*}$ & $91.5(87.5-95.5)$ \\
Overall agreement & 90.1 (86.0-94.2)
\end{tabular}

Abbreviations: $\mathrm{Cl}$ = confidence interval; $\mathrm{EIA}=$ enzyme immunoassay; STD = sexually transmitted disease.

* Sensitivity and specificity were calculated comparing the results of the Syphilis Health Check against the reference treponemal tests used at the state public health laboratory in Florida. 


\footnotetext{
${ }^{1}$ Division of STD Prevention, National Center for HIV/AIDS, Viral Hepatitis, STD, and TB Prevention, CDC; ${ }^{2}$ Florida Department of Health; ${ }^{3}$ Florida Department of Health in Escambia County; ${ }^{4}$ Florida Department of Health, Bureau of Public Health Laboratories.

Corresponding Author: James Matthias, lnk1@cdc.gov, 850-245-4308.
}

\section{References}

1. Food and Drug Administration. FDA grants CLIA waiver expanding the availability of rapid screening test for syphilis. FDA News Release. December 15, 2014. Washington, DC: US Department of Health and Human Services, Food and Drug Administration; 2014. http://www.fda. gov/NewsEvents/Newsroom/PressAnnouncements/ucm426843.htm

2. CDC. Sexually transmitted disease surveillance 2015. Atlanta, GA: U.S. Department of Health and Human Services, CDC; 2016. http://www. cdc.gov/std/default.htm

3. Diagnostics Direct. Syphilis Health Check Test. $510(\mathrm{k})$ summary. August 1, 2011 Stone Harbor, NJ: Diagnostics Direct; 2011. http://www. accessdata.fda.gov/cdrh_docs/pdf10/k102400.pdf 